\title{
Controle Ótimo de Pragas com Base no Modelo Generalizado de Presa-Predador
}

\author{
M. RAFIKOV, E.R. MALEICO, Programa de Pós-Graduação em Modelagem \\ Matemática, DEFEM, UNIJUI, Cx.P.560, 98700-000 Ijuí, Brasil.
}

Resumo. Neste trabalho foi formulado o problema de controle ótimo para sistemas generalizados do tipo presa - predador, com as funções de controle que entram nestes modelos em forma não-linear. Esse problema foi resolvido através da aplicação do Princípio do Máximo de Pontryagin.

\section{Introdução}

Muitos métodos de controle de pragas na agricultura são baseados em inseticidas químicos. As desvantagens destes inseticidas são [3]: a) redução da eficiência devido à resistência dos insetos aos inseticidas utilizados; b) alto impacto direto sobre os inimigos naturais; c) redução da eficiência do controle natural pela brusca redução de seus hospedeiros; d) ressurgência de pragas; e) surgimento de pragas secundárias ou novas pragas; f) presença de resíduos nos alimentos; g) acidentes ambientais; h) alta persistência no ambiente; i) elevado número de acidentes causando intoxicação e/ou óbito dos operadores. Sabendo-se que a poluição por inseticidas causa estas desvantagens, isso levantou interesse cada vez mais crescente no controle biológico de insetos e plantas pragas. O controle biológico é um processo de supressão de populações através de seus inimigos naturais. Estes utilizam o inseto-praga como alimento, reduzindo o seu potencial biótico. O controle biológico é raramente empregado sozinho. Na maioria das vezes, é usado em associação a outros métodos compatíveis de controle como, por exemplo, a aplicação racional de inseticidas não persistentes; mensageiros químicos, como os hormônios e variedades de plantas parcialmente resistentes ao ataque de pragas. Essa abordagem ampla de controle múltiplo de pragas é denominada de manejo integrado de pragas. Este manejo visa reduzir a degradação do meio ambiente e aumentar a produtividade da lavoura. Geralmente, no controle biológico de pragas, os parasitóides ou predadores são importados ou criados em laboratório. Quando há um número adequado de inimigos naturais, eles são lançados com a expectativa da criação de um sistema presa-predador ou hospedeiro-parasita estável. O conhecimento do ciclo biológico, dos hábitos e da dinâmica populacional das culturas, das pragas e de seus inimigos naturais constitui a base do manejo de pragas e determina o sucesso ou o fracasso da utilização do controle biológico de insetos-praga. A escolha mal feita do tipo de inseticida, do momento de aplicação ou do uso de práticas agrícolas prejudiciais 
aos agentes do controle biológico, podem favorecer a ressurgência de pragas e de populações elevadas de insetos fitófagos secundários nas lavouras, provocando, em conseqüência, um aumento no custo de produção devido à necessidade de adoção de novas medidas de controle [2]. Um dos caminhos mais importantes em que a matemática é usada no controle de pragas é formular uma boa estratégia do controle através da manipulação dinâmica de variáveis de controle do sistema praga - inimigo natural. Devido a dificuldades na aplicação da teoria do controle ótimo, existem poucos trabalhos sobre a matemática no controle de pragas $[4,1,8]$. Goh usou a teoria de controle ótimo para formular uma estratégia ótima para alguns modelos simples [5]. Nestes modelos as variáveis de controle são taxas de aplicação de inseticidas químicos ou taxas de lançamento de predadores. Estas variáveis entram no critério de otimização e nas equações em forma linear. Por exemplo, foi considerado por Goh um problema de otimização do lançamento de predadores [5]. O modelo do controle do sistema presa - predador é

$$
\frac{d x}{d t}=x(1-y), \quad \frac{d y}{d t}=y(x-1)+u,
$$

onde $x$ e $y$ são as populações de presas e predadores, respectivamente, e $u$ é a taxa de lançamento de predadores que foram criados em laboratórios ou importados. As condições iniciais são:

$$
x(0)=x_{0}, \quad y(0)=y_{0} .
$$

As condições finais são:

$$
x(T)=1, \quad y(T)=1,
$$

onde $T$, tempo final, não é limitado. As limitações da função de controle são:

$$
0 \leq u(t) \leq b,
$$

onde b é uma constante positiva. O critério de otimização é:

$$
J=T+\int_{0}^{T}\left(c_{1} x+c_{2} y\right) d t
$$

onde $c_{1}$ e $c_{2}$ são constantes não-negativas.

O problema é encontrar uma função admissível $u^{*}(t)$ que transfira o sistema (1.1) do estado inicial (1.2) ao estado final (1.3), e que minimize o funcional (1.5). Geralmente, no controle de pragas, grande quantidade de espécies são removidas do sistema durante o período de aplicação. Estas espécies não participam mais do processo reprodutivo, competitivo e de interação. Ao contrário, as espécies de predadores são introduzidas no sistema e elas começam imediatamente a participar do processo de reprodução, competição e predação. A introdução da variável de controle $u(t)$ no problema (1.1) - (1.5) é equivalente ao aumento da taxa de natalidade da população predatória no instante $t$. De acordo com o modelo referido acima, este controle não influencia a competição e a predação no instante $t$. 


\section{Formulação do problema}

Consideremos o modelo presa-predador:

$$
\begin{aligned}
& \frac{d x}{d t}=x f(x, y), \\
& \frac{d y}{d t}=y g(x, y),
\end{aligned}
$$

onde $x$ e $y$ são as densidades de presas e predadores, respectivamente, no instante $t$ e $f(x, y)$ e $g(x, y)$ são funções contínuas das variáveis $x$ e $y$. O sistema (2.1) descreve o desenvolvimento natural do sistema presa-predador sem a aplicação do controle. Sejam $U(t)$ e $V(t)$ o número de presas retiradas do sistema e o número de predadores introduzidos no sistema no instante $t$, respectivamente. As equações que descrevem a dinâmica do sistema com a aplicação do controle podem ser escritas na forma seguinte:

$$
\begin{aligned}
& \frac{d x}{d t}=(x-U) f(x-U, y+V)-k_{1} U \\
& \frac{d y}{d t}=(y+V) g(x-U, y+V)+k_{2} V
\end{aligned}
$$

onde $k_{1}$ e $k_{2}$ são constantes positivas que caracterizam as condições técnicas de aplicação de controle. As funções de controle $U(t)$ e $V(t)$ satisfazem às limitações:

$$
\begin{aligned}
& 0 \leq U \leq x, \\
& 0 \leq V
\end{aligned}
$$

Suponha que seja desejável segurar o nível de pragas abaixo dos danos econômicos para pragas prejudiciais e ter um baixo custo no uso da variável de controle. Para atingir estes objetivos, usamos o critério de otimização

$$
I=c_{1}\left[x(T)+k_{1} \int_{t_{0}}^{T} U(t) d t\right]+c_{2}\left[k_{2} \int_{t_{0}}^{T} V(t) d t-y(T)\right]
$$

onde $c_{1}$ e $c_{2}$ são constantes positivas que caracterizam a influência de cada tipo de controle e $t_{0}$ e $T$ são os momentos inicial e final da aplicação do controle, respectivamente. Minimizando o critério (2.4), estamos minimizando os valores das funções de controle e da população de pragas e maximizando a população de predadores.

O problema do controle ótimo consiste em escolher um programa de controle admissível, que levará o sistema (2.2) do estado inicial

$$
x(0)=x_{0}, \quad y(0)=y_{0},
$$

para o estado final, tal que o critério (2.4) seja minimizado. Este problema de otimização de um sistema dinâmico pode ser resolvido através do Princípio do Máximo (Pontryagin et al., 1962). 


\section{Resultados e conclusões}

Para facilitar, introduzimos uma nova variável $w$,

$$
w(t)=c_{1}\left[x(t)+k_{1} \int_{t_{0}}^{t} U(t) d t\right]+c_{2}\left[k_{2} \int_{t_{0}}^{t} V(t) d t-y(t)\right],
$$

cuja derivada é

$$
\frac{d w}{d t}=c_{1}\left[\frac{d x}{d t}+k_{1} U(t)\right]+c_{2}\left[k_{2} V(t)-\frac{d y}{d t}\right] .
$$

Substituindo as equações (2.2) na expressão acima, temos:

$$
\frac{d w}{d t}=c_{1}(x-U) f(x-U, y+V)-c_{2}(y+V) g(x-U, y+V) .
$$

Denotaremos

$$
\begin{gathered}
\zeta_{1}=x-U(t), \\
\zeta_{2}=y+V(t) .
\end{gathered}
$$

Vamos definir a função de Hamilton

$$
\begin{aligned}
H= & \psi_{0}\left[c_{1} \zeta_{1} f\left(\zeta_{1}, \zeta_{2}\right)-c_{2} \zeta_{2} g\left(\zeta_{1}, \zeta_{2}\right)\right] \\
& +\psi_{1}\left[\zeta_{1} f\left(\zeta_{1}, \zeta_{2}\right)-k_{1} U(t)\right]+\psi_{2}\left[\zeta_{2} g\left(\zeta_{1}, \zeta_{2}\right)+k_{2} V(t)\right],
\end{aligned}
$$

onde $\psi_{0}, \psi_{1}$ e $\psi_{2}$ são variáveis conjugadas determinadas pelas seguintes equações:

$$
\begin{aligned}
\frac{d \psi_{0}}{d t} & =-\frac{\partial H}{\partial w}=0, \\
\frac{d \psi_{1}}{d t} & =-\frac{\partial H}{\partial x}, \\
\frac{d \psi_{2}}{d t} & =-\frac{\partial H}{\partial y} .
\end{aligned}
$$

e condições finais:

$$
\begin{aligned}
& \psi_{0}(T)=-1 \\
& \psi_{1}(T)=0 \\
& \psi_{2}(T)=0 .
\end{aligned}
$$

Conforme o Princípio do Máximo, as funções de controle ótimo maximizam a função $H$. As condições necessárias para o máximo da função $H$ são: 


$$
\begin{aligned}
& \frac{\partial H}{\partial U}=-\frac{\partial H}{\partial \zeta_{1}}-k_{1} \psi_{1}=0 \\
& \frac{\partial H}{\partial V}=\frac{\partial H}{\partial \zeta_{2}}+k_{2} \psi_{2}=0 .
\end{aligned}
$$

Da primeira equação do sistema (3.6), temos:

$$
\psi_{0}=\text { const }
$$

Aplicando a primeira condição final (3.7), obtemos:

$$
\psi_{0}=-1
$$

Da primeira equação do sistema (3.8), temos:

$$
\frac{\partial H}{\partial \zeta_{1}}=-k_{1} \psi_{1} .
$$

Por outro lado,

$$
\frac{\partial H}{\partial x}=\frac{\partial H}{\partial \zeta_{1}}
$$

conseqüentemente, a segunda equação do sistema (3.6) pode ser escrita como

$$
\frac{d \psi_{1}}{d t}=k_{1} \psi_{1}
$$

A solução geral da equação (3.10) é

$$
\psi_{1}=A e^{k_{1} t}
$$

Aplicando a segunda condição final (3.7) obtemos $A=0$ e, conseqüentemente,

$$
\psi_{1}=0
$$

Para a terceira equação do sistema (3.6), por análise similar, obtemos:

$$
\psi_{2}=0 \text {. }
$$

Aplicando (3.9), (3.11) e (3.12) em (3.5), obtemos:

$$
H=-c_{1} \zeta_{1} f\left(\zeta_{1}, \zeta_{2}\right)+c_{2} \zeta_{2} g\left(\zeta_{1}, \zeta_{2}\right) .
$$

Agora, a partir de (3.8), obtemos o sistema de duas equações

$$
\begin{gathered}
c_{1} f\left(\zeta_{1}, \zeta_{2}\right)+c_{1} \zeta_{1} \frac{\partial f}{\partial \zeta_{1}}-c_{2} \zeta_{2} \frac{\partial g}{\partial \zeta_{1}}=0 \\
-c_{1} \zeta_{1} \frac{\partial f}{\partial \zeta_{2}}+c_{2} g\left(\zeta_{1}, \zeta_{2}\right)+c_{2} \zeta_{2} \frac{\partial g}{\partial \zeta_{2}}=0 .
\end{gathered}
$$


Utilizando (2.3), (3.4) e os valores de $\zeta_{1}$ e $\zeta_{2}$ calculados das equações (3.14) e (3.15), obtemos:

$$
\begin{aligned}
& U(t)=\left\{\begin{array}{rll}
x-\zeta_{1} & \text { quando } & x>\zeta_{1} \\
0 & \text { quando } & x \leq \zeta_{1}
\end{array}\right. \\
& V(t)=\left\{\begin{array}{rrr}
\zeta_{2}-y & \text { quando } & y<\zeta_{2} \\
0 & \text { quando } & y \geq \zeta_{2}
\end{array}\right.
\end{aligned}
$$

Consideremos o modelo clássico Lotka - Volterra. Neste caso, temos:

$$
\begin{aligned}
& f(x, y)=a-\alpha y, \\
& g(x, y)=-b+\beta x .
\end{aligned}
$$

As equações (3.14) e (3.15) podem ser escritas como

$$
\begin{aligned}
c_{1}\left(a-\alpha \zeta_{2}\right)-c_{2} \beta \zeta_{2} & =0 \\
c_{1} \alpha \zeta_{1}+c_{2}\left(-b+\beta \zeta_{1}\right) & =0 .
\end{aligned}
$$

Resolvendo (3.18), obtemos:

$$
\begin{aligned}
\zeta_{2} & =\frac{c_{1} a}{c_{1} \alpha+c_{2} \beta}, \\
\zeta_{1} & =\frac{c_{2} b}{c_{1} \alpha+c_{2} \beta} .
\end{aligned}
$$

Para ilustrar a aplicação da metodologia proposta para o controle ótimo de pragas, foi considerada a relação presa-predador entre lagarta da soja (Anticarsia gematalis) e seus predadores (Nabis spp, Geocoris, Aracnideo, etc.). Os coeficientes do modelo foram encontrados no trabalho [7]. Neste caso, os coeficientes do modelo são: $a=0,216 ; \quad \alpha=0,0108 ; \quad b=0,173 ; \quad \beta=0,0029 ; \quad c_{2}=1,7 ; \quad c_{1}=k_{1}=$ $k_{2}=1$. Na Fig.1 o diagrama de fase para o problema do controle ótimo é dado para todos os estados iniciais positivos do sistema considerado (2.2) e (3.17). As variáveis de controle nesta maneira compõem uma estratégia ótima do controle dada por (3.16).

As retas $\zeta_{1}=18,68$ e $\zeta_{2}=13,73$ na Fig.1 são linhas de comutação. Elas dividem o quadrante positivo em quatro regiões. Para qualquer trajetória que tenha o estado inicial na região $A$, as funções de controle são nulas, isto é, $U=0$ e $V=0$ até que sua solução intercepte a reta de comutação $\zeta_{2}$. No ponto de interseção a variável de controle $V$ torna-se positiva e a função $U$ permanece nula enquanto a trajetória fica na região $D$. No ponto de interseção com a reta $\zeta_{2}$ a variável de controle $U$ torna-se positiva. Na região $C$ as duas funções de controle permanecem ligadas até que a trajetória do sistema chegue ao ponto de equilíbrio $P$ em $(19,94,12,1)$. Quando o estado inicial do sistema está em $B$, a variável de controle $V$ é nula e $U$ é positiva 


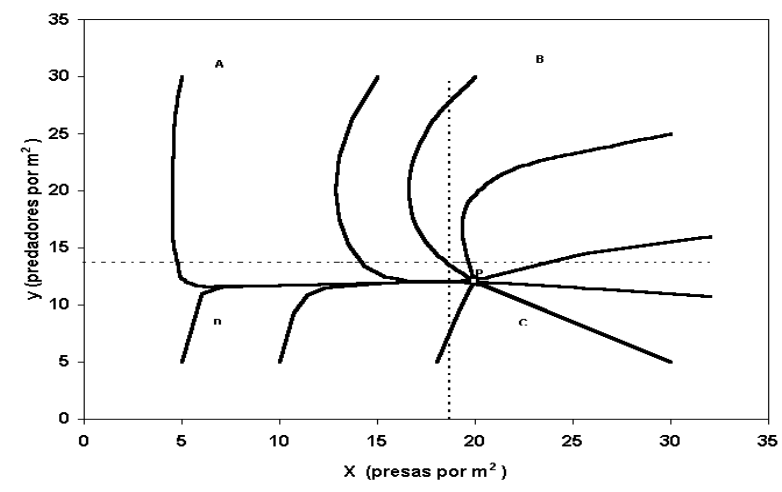

Figura 1: Diagrama de fase para o problema do controle ótimo com parâmetros do modelo $a=0,216 ; \quad \alpha=0,0108 ; \quad b=0,173 ; \quad \beta=0,0029 ; \quad c_{1}=c_{2}=k_{1}=$ $k_{2}=1$.

até que a solução intercepte a linha de comutação $\zeta_{1}$ ou $\zeta_{2}$. Ao interceptar $\zeta_{1}$, a variável de controle $U$ é desligada e o sistema envolve sua própria dinâmica natural até que a trajetória intercepte a reta de comutação $\zeta_{2}$. Então a variável de controle $U$ é ligada novamente e isto leva o sistema para o ponto de equilíbrio $P$. Na Fig.2 é apresentada a trajetória que caracteriza a variação de densidade de populações de pragas ao longo do tempo para condições iniciais $x(0)=15$ e $y(0)=30$. A parte inicial da trajetória do sistema com controle (curva cheia) coincide com a trajetória do desenvolvimento natural (curva tracejada).

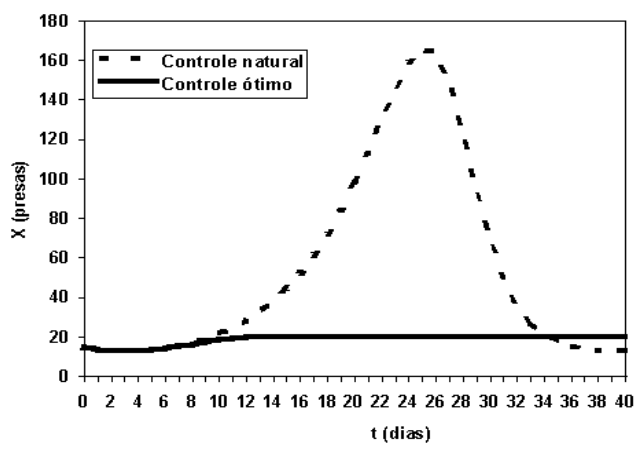

Figura 2: Densidade de população de pragas ao longo do tempo para condições inicias $x(0)=15$ e $y(0)=30$

A Fig. 3 apresenta a variação de densidade de populações de predadores ao longo do tempo. 


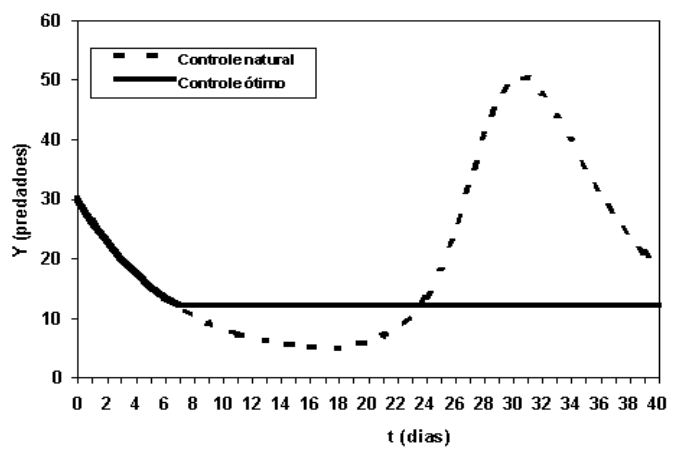

Figura 3: Densidade de população de predadores ao longo do tempo para condições inicias $x(0)=15$ e $y(0)=30$.

\section{Agradecimentos}

Um dos autores agradece FAPERGS pelo apoio ao trabalho.

\section{Referências}

[1] G.R. Conway, Mathematical Models in Applied Ecology, Nature, 269 (1977), 291-297.

[2] D.N. Gassen, "Parasítas, patógenos e predadores de insetos associados à cultura do trigo", Passo Fundo, EMBRAPA-CNPT, 1986.

[3] D.L. Gazzoni, "Manejo de pragas da soja: uma abordagem histórica", Londrina, EMBRAPA-CNPSO, 1994.

[4] B.S. Goh, G. Leitmann and T.L. Vincent, The Optimal Control of a PreyPredator System, Math. Biosci., 19 (1974), 263-286.

[5] B.S. Goh, "Management and Analysis of Biological Populations", Elsevier Scientific Publishing Company, Amsterdam, Oxford, New York, 1980.

[6] L.S. Pontryagin, V.G. Boltyanskii, R.V. Gamkrelidze and E.F. Mischenko, "The Mathematical Theory of Optimal Processes", New York, Interscience Publishers, Inc., 1962.

[7] M. Rafikov, Determinação dos parâmetros de modelos biomatemáticos, Ciência e Natura, 19 (1997), 7-20.

[8] C.A. Shoemaker, "Ecosystem Modeling in Theory and Practice", (C.A.S. Hall and J.W. Day, eds.), John Wiley and Sons, New York, pp. 546-574, 1977. 